\title{
A!
}

This is an electronic reprint of the original article.

This reprint may differ from the original in pagination and typographic detail.

I-Kai Wang, Kevin; Zhou, Xiaokang; Liang, Wei; Yan, Zheng; She, Jinhua

\section{Federated Transfer Learning Based Cross-Domain Prediction for Smart Manufacturing}

Published in:

IEEE Transactions on Industrial Informatics

DOI:

10.1109/TII.2021.3088057

Published: 01/06/2022

Document Version

Peer reviewed version

Please cite the original version:

I-Kai Wang, K., Zhou, X., Liang, W., Yan, Z., \& She, J. (2022). Federated Transfer Learning Based CrossDomain Prediction for Smart Manufacturing. IEEE Transactions on Industrial Informatics, 18(6), 4088-4096. https://doi.org/10.1109/TII.2021.3088057

This material is protected by copyright and other intellectual property rights, and duplication or sale of all or part of any of the repository collections is not permitted, except that material may be duplicated by you for your research use or educational purposes in electronic or print form. You must obtain permission for any other use. Electronic or print copies may not be offered, whether for sale or otherwise to anyone who is not an authorised user. 
(C) 2021 IEEE. This is the author's version of an article that has been published by IEEE. Personal use of this material is permitted. Permission from IEEE must be obtained for all other uses, in any current or future media, including reprinting/republishing this material for advertising or promotional purposes, creating new collective works, for resale or redistribution to servers or lists, or reuse of any copyrighted component of this work in other works. 


\title{
Federated Transfer Learning Based Cross-Domain Prediction for Smart Manufacturing
}

\author{
Kevin I-Kai Wang, Member, IEEE, Xiaokang Zhou, Member, IEEE, Wei Liang, Member, IEEE, Zheng \\ Yan, Senior Member, IEEE, and Jinhua She, Fellow, IEEE
}

\begin{abstract}
Smart manufacturing aims to support highly customizable production processes. Therefore, the associated machine intelligence needs to be quickly adaptable to new products, processes, and applications with limited training data, while preserving data privacy. In this paper, a new federated transfer learning framework, FTL-CDP (Federated Transfer Learning for Cross-Domain Prediction), is proposed to address the challenges of data scarcity and data privacy faced by most machine learning approaches in modern smart manufacturing with cross-domain applications. The framework architecture consists of a central server and several groups of smart devices, where each group handles a different application. Existing applications can share their knowledge through the central server as base models, while new applications can convert a base model to their target domain models with limited application-specific data using transfer learning technique. Meanwhile, the federated learning scheme is deployed within a group to further enhance the accuracy of the application-specific model. The integrated framework allows model sharing across the central server and different smart devices without exposing any raw data, and hence protects data privacy. Two public datasets COCO and PETS2009, which represent the source and target applications, are employed for evaluations. The simulation results show that the proposed method outperforms two state-of-the-art machine learning approaches, by achieving better learning efficiency and accuracy.
\end{abstract}

Index Terms - Federated Learning, Transfer Learning, Internet of Things, Cross-Domain Prediction, Industry 4.0

\section{INTRODUCTION}

$\mathrm{T}$ he advancement of embedded and communication technologies has driven the rapid development of Industry 4.0 to allow more efficient and customizable production and

Kevin Wang is with the Department of Electrical, Computer, and Software Engineering, The University of Auckland, Auckland 1010, New Zealand (e-mail: kevin.wang@auckland.ac.nz).

Xiaokang Zhou (corresponding author) is with the Faculty of Data Science, Shiga University, Hikone 522-8522, Japan, and also with the RIKEN Center for Advanced Intelligence Project, RIKEN, Tokyo 103-0027, Japan (e-mail: zhou@biwako.shiga-u.ac.jp).

Wei Liang (corresponding author) is with the Base of International Science and Technology Innovation and Cooperation on Big Data Technology and Management, Hunan University of Technology and Business, Changsha 410205, China (e-mail: weiliang@csu.edu.cn).

Zheng Yan is with the State Key Laboratory on Integrated Services Networks and the School of Cyber Engineering, Xidian University, Xi'an 710071, China, and Department of Communications and Networking, Aalto University, Espoo 02150, Finland (e-mail: zyan@xidian.edu.cn).

Jinhua She is with the School of Engineering, Tokyo University of Technology, Tokyo 192-0982, Japan (e-mail: she@stf.teu.ac.jp). logistic processes. Empowered by extensive sensing and machine intelligence, industrial applications are becoming increasingly data-driven. With the fast changing and evolving modern manufacturing and warehousing processes, machine intelligence needs to be able to adapt quickly to different applications. While existing deep learning techniques are able to deliver good results with sufficient training data, the required dataset and learning time still present major obstacles. Therefore, such techniques are not easily applicable across different knowledge domains and new applications [1-4]. In addition, the collected data may contain confidential information and hence data sharing may not be possible even within the same enterprise, which further limits the potential of modern machine intelligence in industrial applications $[5,6]$.

The distributed nature of modern Industrial Internet of Things (IIoT) systems together with traditional centralized machine learning techniques poses immediate threats to data sharing. Considering object/human recognition in smart manufacturing as an example, raw data collected from different cameras needs to be uploaded to a centralized server (e.g., a cloud server). This may result in the potential leakage of confidential information, for instance, of the product being manufactured or unnecessary personal information being collected. The concept of federated learning is recently introduced to facilitate knowledge sharing across multiple smart end devices while protecting data privacy by avoiding raw data to be shared across end devices and a central server [7-10]. In federated learning based smart manufacturing systems, multiple cameras can collect product images and train a local model individually. The local model parameters, instead of raw data, can be encrypted and uploaded to a central server for the formation of a global model. By combining multiple local models, the global model is expected to provide a more generalized and accurate predictions.

In addition to data privacy, it is also crucial to address the challenge of knowledge sharing and data scarcity across different domains and heterogeneous applications. For example, in a modern warehouse or a production line setting, there may be existing datasets and trained models for certain object detection (e.g., for the purpose of pallet detection). It would be highly beneficial, if the trained model and its domain knowledge can be applied as a starting point for other automation applications, such as product identification and worker detection, to overcome the limitation of data scarcity.

Although federated learning protects data privacy within one 
application, the amount of data and time required during training processes may still remain high. The challenge of data scarcity is likely to exist in many applications, if not all. While modern and future industrial applications are targeting high flexibility and customizability, commonalities among multiple applications should be exploited and utilized to enhance machine intelligence across different domains and applications. The concept of transfer learning can be applied to make use of an existing trained model for different target applications to speed up the training process with less data [11].

In this paper, a new Federated Transfer Learning framework for Cross-Domain Prediction (FTL-CDP) is proposed targeting cross-domain applications in smart manufacturing, which have limited training data and need to ensure data privacy. The FTL-CDP framework combines the concepts of federated learning and transfer learning and takes benefits from both sides to address the challenges of data scarcity and privacy in achieving machine intelligence in Industry 4.0 environments. It supports distributed training that protects the data privacy, while allowing trained model to be exchanged across different domains, such as object detection and pedestrian detection, to speed up the training process with limited amount of data. The main contributions of this paper are as follows.

i) A new framework architecture targeting modern Industry 4.0 scenario is designed, which can be integrated with existing smart manufacturing systems, warehouse/logistic control systems, and enterprise systems.

ii) To the best of our knowledge, this is the first federated transfer learning framework proposed for cross-domain heterogeneous applications. The proposed framework is able to achieve a more accurate target application model using less training data and training time, while protecting data privacy across multiple smart devices

iii) The proposed framework is evaluated using two public datasets (COCO and PETS2009) to investigate and demonstrate its ability in handling cross-domain knowledge transfer, and the strength in achieving more efficient and accurate learning.

The rest of this article is organized as follows. Section II provides an overview of related works on existing federated and transfer learning, and their associated challenges. The motivating scenario and the overall framework architecture is presented in Section III, followed by the detailed methodologies explained in Section IV. Section V describes our detailed experimental setup and results, involving an object and pedestrian detection scenarios using the proposed method. We conclude this study and give our promising perspectives regarding future research in Section VI.

\section{RELATED WORK}

In this section, current studies related to federated learning, transfer learning, and federated transfer learning are discussed.

\section{A. Trends on Federated Learning}

In recent years, federated learning has drawn a widespread attention as a privacy-preserving distributed learning technique. This is primarily due to the increasing privacy concerns when dealing with large amounts of user-generated data for detection and prediction tasks. Song et al. [12] focused on user-level privacy issue, and presented an attack framework based on attacks from malicious servers. An anonymization strategy was designed to update the model from different clients. Lyu et al. [13] considered the issue on collaborative fairness in traditional federated learning model. They proposed a mechanism based on local credibility for fairness evaluation, and designed a three-layer encryption scheme for both privacy and accuracy guarantee. Zhu and Jin [14] viewed federated learning as a bi-objective optimization problem to minimize the communication cost and maximize the global learning accuracy. Based on the improvement of neural network structure, they developed an evolutionary algorithm to reduce errors in the global model, and proposed a scalable method to optimize the encoding network connectivity. Lu et al. [15] built a blockchain based framework for data sharing with multiple parties, in which the federated learning model was utilized to preserve the privacy in industrial IoT environments. Hao et al. [16] constructed a privacy-enhanced federated learning to deal with the privacy leakage using local gradients. They proposed a homomorphic encryption scheme to reduce the key-switching operation, and employed a distributed Gaussian mechanism to enhance privacy protection in training data.

As an emerging technique, existing researches on federated learning mainly focus on privacy preservation across multiple clients or IoT devices. Some of them aim to enhance algorithm performance in terms of model accuracy and communication efficiency [17]. Different to the existing works, this study explores the challenge of federated learning in terms of its learning data efficiency and accuracy in cases of supporting heterogeneous applications with limited training data. This is essential to address the challenge in modern manufacturing and warehousing processes, which are highly flexible and may involve several parallel automated systems offering different services or productions. Such applications are likely to have only a small amount of training data, but require efficient learning algorithms to quickly adapt in a short period of time.

\section{B. Trends on Transfer Learning}

Comparing with other machine learning methods, transfer learning is a typical learning technique to transfer a domain knowledge into a new domain, and reduce the time-consuming data-labeling cost [11]. Fang et al. [18] discussed a cross-network transfer learning model, to identify the subgraph patterns between the source and target network, which might improve the classification accuracy in the target network. They extracted structure features, and transferred them to generate new features in the target network based on an iterative classification algorithm. Sun et al. [19] introduced a sparse autoencoder based deep transfer learning framework, in which the historical failure data was utilized for the weight transfer and update. The learned features were employed to improve the prediction accuracy in a case study based experiment. Considering homogeneous transfer learning and heterogeneous transfer learning in an online transfer learning problem, $\mathrm{Wu}$ et al. [20] designed an algorithm based on multi-source domains. 
According to the theoretical analysis, the method demonstrated the ability to achieve a higher classification accuracy by using labeled data from multiple sources. Li et al. [21] introduced a deep learning based diagnostics method, in which the transfer learning was integrated with domain adversarial training. Based on their method, the diagnostic knowledge could be learned from supervised data with different label spaces of multiple rotating machines, and transferred to the target equipment. Yang et al. [22] analyzed the principal components in each feature space, to learn the transferred weights based on co-occurrence data. They constructed a directed cyclic network based on a Markov Chain Monte Carlo method, in which the principal component analysis technique was used to analyze the principal component coefficients from different feature spaces.

\section{Trends on Federated Transfer Learning}

While federated learning allows privacy-preserving knowledge sharing, the global model is generic and may not offer optimal results for every individual device. Transfer learning, on the other hand, allows knowledge from one domain to be used in solving a problem in a different domain. Although these two approaches naturally complement each other, there are only a few works based on federated transfer learning, which can be categorized into two directions.

The first direction shares a similar goal as existing federated learning research, focusing mainly on ensuring privacy protection while performing domain knowledge transformation in distributed systems. Liu et al. [23] presented a secure protocol for federated transfer learning that allowed domain knowledge transfer across two participants using homomorphic encryption. Sharma et al. [24] proposed a different protocol that made use of secure multi-party, which improved the algorithm efficiency by an order of magnitude.

The second direction focuses more on the applicability of federated transfer learning in offering personalized models while achieving privacy-preserving knowledge sharing. Existing works mainly focused on healthcare applications which involved sensitive personal data that cannot be shared with a central server. At the same time, there is also the need to have personalized models to offer suitable healthcare services. Chen et al. [25] proposed a federated transfer learning framework to be trained on wearable sensor data. The federated learning adopted typical convolutional neural network (CNN) with feature-based transfer learning, where the convolution layers were shared between individual user models and the server model. Ju et al. [26] proposed a federated transfer learning framework for EEG signal classification. A neural network was developed using the covariance matrix of EEG signals. Federated learning was adopted to allow data sharing cross multiple devices and transfer learning was used to achieve subject-specific classification. In both cases, the proposed federated transfer learning frameworks target the same application with different data distributions across different human subjects.

In this study, a new framework that combines the federated learning and transfer learning is designed to support cross-domain heterogeneous smart manufacturing applications.
The federated learning enables privacy protected model sharing across multiple devices, while transfer learning allows individual devices to develop application-specific models with less training data and faster convergence.

\section{FEDERATED TRANSFER LEARNING FRAMEWORK FOR CROSS-DOMAIN SMART MANUFACTURING APPLICATIONS}

In this section, a motivating scenario is first presented targeting heterogeneous smart manufacturing applications. An overview of the proposed federated transfer learning framework is then introduced and discussed.

\section{A. Motivating Scenario}

In modern Industry 4.0 and smart manufacturing applications, enterprise level management, production line automation, and warehouse/logistic control systems are all interconnected. However, the entire process also needs to be highly flexible to support customizable products, reconfigurable automation/production processes, and better securities. These heterogeneous applications demand modern machine intelligence to be smarter to cope with the fast-changing reality. In this study, we aim to propose a new federated transfer learning framework that facilitates the knowledge sharing and training with limited data for heterogeneous smart manufacturing applications.

Fig. 1 illustrates a typical Industry 4.0 scenario which consists of a production line monitoring system, a building security system, and a warehouse monitoring system. Different applications are established at different times, with different levels of robustness, and are trained with varying amount of training data. For example, the building security system may be trained with a large amount of data to ensure good reliability. However, a warehouse that is subdivided for different products with different workers and machineries (e.g., forklifts) may face a more dynamic application scenario. To establish a new application to recognize a new product or to detect workers for a safe working zone in a warehouse, there may not be sufficient data to train a new model. Therefore, a federated transfer learning framework is proposed to facilitate the training process of new heterogeneous applications using existing models with privacy preservation. As shown in Fig. 1, within an enterprise, there can be several existing models trained for various purposes such as object detection or facial recognition. These existing models may contain transferrable knowledge that can help to establish new applications quickly.

At the initialization stage, smart devices of a new application will request the central server for a base model. The central server will search and select a suitable base model (i.e., with good similarity measure), and distribute the model to the smart devices. The smart devices, upon receiving the base model, will apply transfer learning using their local data to build application-specific models. These local models will then be fed back to the central server for model aggregation among the group of devices targeting the same application, following the federated learning paradigm. The aggregated global model will be redistributed to the relevant devices iteratively to improve the overall learning performance. 


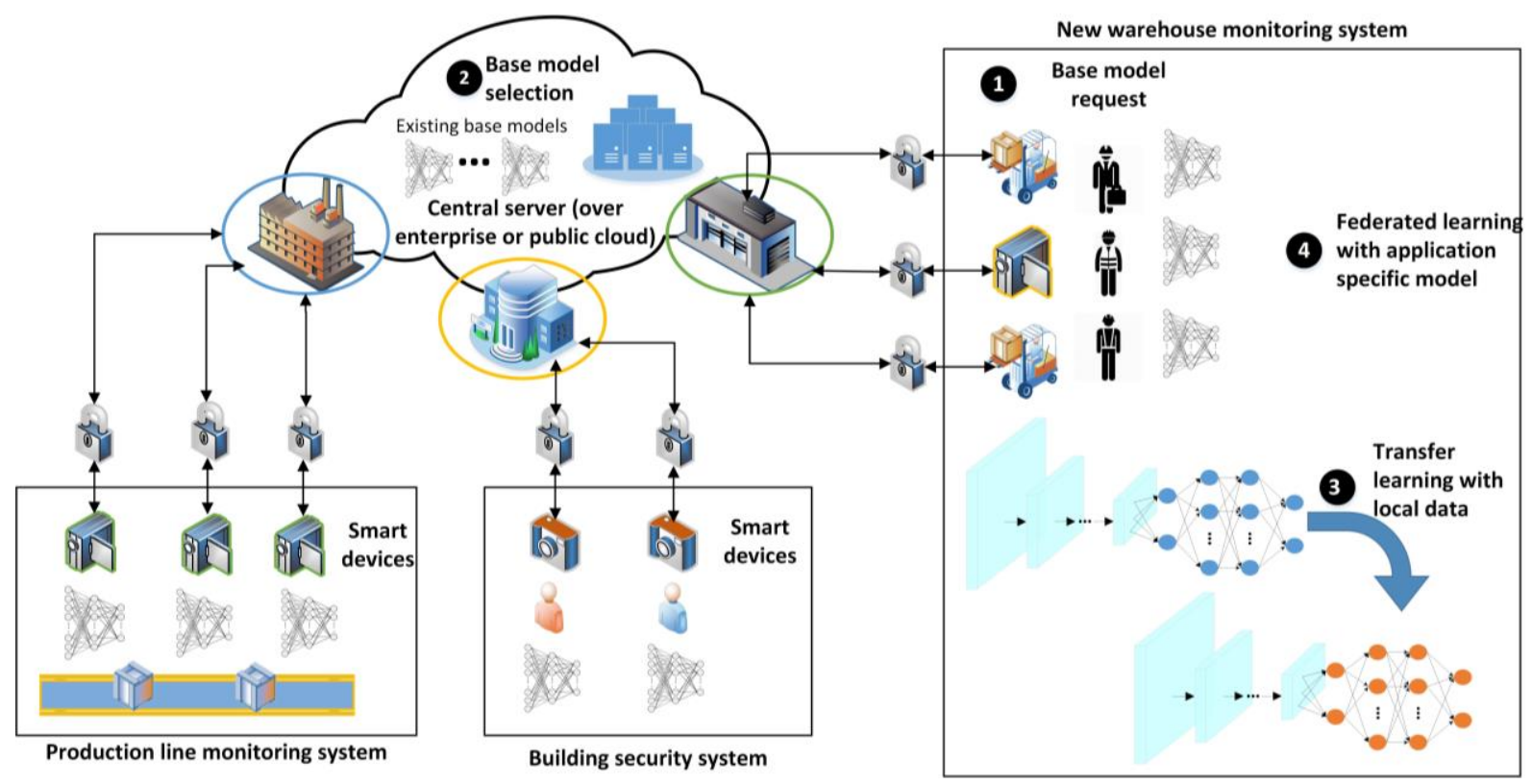

Fig. 1. The federated transfer learning for cross-domain prediction (FTL-CDP) framework architecture.

\section{B. Federated Transfer Learning Framework Architecture}

Based on the motivating scenario, the FTL-CDP framework architecture is designed to fit with a typical Industry 4.0 system infrastructure. As shown in Fig. 1, the architecture consists of a central server and several smart devices belonging to different applications. The central server is responsible for managing existing base models, which could be acquired through external sources, or internally trained within an enterprise. The existing base models are generated from existing applications such as object or worker detection possibly in different environments, or for different applications, such as factory or warehouse monitoring. Smart devices are application-specific and can be deployed for various purposes, for instance, product identification, employee tracking, or logistic monitoring. While smart devices constantly collect data, the collected data may not be sufficient to train a proper model starting from scratch.

In the proposed architecture, transfer learning is applied in each smart device to speed up the learning process by making use of an existing base model to address the challenge of data scarcity for heterogeneous applications. However, it is important even within the same enterprise to avoid unnecessary product or personnel information leaking. Therefore, the federated learning paradigm is adopted to ensure privacy protection across multiple devices. Referring to Fig. 1, it is clear that individual smart devices will not need to share data with each other. Model parameters shared with the central server are encrypted following the horizontal federated learning paradigm, thus the data privacy is protected. Overall, the proposed framework can facilitate machine intelligence applications across different domains in a distributed infrastructure with limited training data.

\section{FEDERATED TRANSFER LEARNING FOR CROSS-DOMAIN PREDICTION}

Extending from the federated transfer learning framework presented in section III, this section first presents the problem definition, followed by the detailed descriptions of the proposed transfer and federated learning integration.

\section{A. Problem Definitions}

Referring to Fig. 1, which illustrates the motivating scenario that consists of $N$ smart devices for different applications such as production line monitoring system, and building security system, and a newly established warehouse monitoring system. These smart devices form a set of data owners $S D=\left\{s d_{i}\right\}_{i=1}^{N}$, which collects real-time application-specific data $D=\left\{d_{i}\right\}_{i=1}^{N}$ that can be used to train a local model. We assume that each local data set $d_{i}$ consists of a set of raw data $x_{i}$ and the corresponding label $y_{i}$ as $d_{i}=\left(x_{i}, y_{i}\right)$. In centralized learning process, all the data is consolidated at the central server. In the proposed framework, a horizontal federated learning architecture is used to manage $M$ heterogeneous applications, each of which consists of a set of $T$ smart devices $S=\left\{s_{j}\right\}_{j=1}^{T}$. As shown in Fig. 1, individual devices start by making requests to the central server when it is responsible for a new application. Based on the similarity, the central server will allocate the most suitable base model of an existing application and share the base model parameters with the corresponding requesting devices. Upon receiving the base model, each individual device performs transfer learning to derive their own application-specific models. Relevant devices belonging to the same application domain will then perform federated learning within their domain to continue the iterative learning process. Individual devices only share their trained local parameters with the central server after encryption. Therefore, data privacy across different smart devices and heterogeneous applications are protected. Iteratively, an application-specific global model, $g_{m}$ (where $G=\left\{g_{m}\right\}_{m=1}^{M}$ ) is trained for each application by aggregating a set of local model parameters learned and uploaded from the relevant group of smart devices. 


\section{B. Cross-Domain Prediction with Transfer Learning Scheme}

While modern industrial applications are highly customizable, there may exist fair amount of commonality across different applications. For example, many low-level features for different computer vision-based applications may be transferrable and may be utilized to speed up the training process. In this paper, we aim to exploit the commonality of cross-domain applications, and make use of transfer learning (or more specifically a domain adaptation technique) to facilitate fast and accurate model training with limited amount of local data collected by individual smart devices.

In a typical deep learning approach such as $\mathrm{CNN}$, different features are extracted in different layers of a neural network. The lower layers extract low level features which are very likely to be transferrable between heterogeneous applications. Therefore, we apply parameter adaptation approach to transfer the base model by updating the associated weights of certain layers using locally collected application-specific data. Given a training sample $d_{i}=\left(x_{i}, y_{i}\right)$, we first define a label classification output computing layer as $G_{y}\left(G_{f}\left(x_{i} ; \theta_{f}\right) ; \theta_{y}\right)$ with parameter $\theta_{y}$, in which $G_{f}\left(x_{i} ; \theta_{f}\right): x_{i} \rightarrow R^{M}$ denotes the function of mapping the original input $x_{i}$ to an $M$-dimensional feature representation $R^{M}$ by the convolutional based feature extraction layer with parameter $\theta_{f}$. The mapping function $G_{f}$ is defined as follows.

$$
G_{f}\left(x_{i}, \theta_{f}\right)=\operatorname{Sigmod}\left(\operatorname{conv}\left(W_{f}, x_{i}\right)+b_{f}\right)
$$

where $\operatorname{Sigmod}(\mathbf{x})=\left[\frac{1}{1+\exp \left(-x_{k}\right)}\right]_{k=1}^{|\mathbf{x}|}, \operatorname{conv}(*)$ denotes the convolution operation, $\theta_{f}=\left(W_{f}, b_{f}\right)$ is the parameter for $G_{f}$.

Considering the cross-domain transfer learning scenario from source domain $D_{S}$ to target domain $D_{T}$, the domain specific loss of a domain logistic regressor $G_{d}\left(G_{f}\left(x_{i}\right)\right)$ is defined in (2) to indicate the influence of the distance between the source and target domain.

$$
\mathcal{L}_{d}^{i}=\mathcal{L}_{d}\left(G_{d}\left(G_{f}\left(x_{i} ; \theta_{f}\right) ; \theta_{y}\right), d m l_{i}\right)
$$

where $d m l_{i}$ stands for a domain label for the sample $d_{i}=$ $\left(x_{i}, y_{i}\right)$, which indicates whether $x_{i}$ come from the source domain $\left(x_{i} \in D_{S}\right.$ if $\left.d m l_{i}=0\right)$ or from the target domain $\left(x_{i} \in D_{T}\right.$ if $\left.d m l_{i}=1\right)$. The domain regressor $G_{d}$ is calculated by a sigmoid function. This loss function is designed to discriminate the feature distribution between the source domain and target domain during the training process. Therefore, the $\mathcal{L}_{d}$ can be defined as [27]:

$$
\mathcal{L}_{d}=d m l_{i} \log \frac{1}{G_{d}\left(G_{f}\left(x_{i}\right)\right)}+\left(1-d m l_{i}\right) \log \frac{1}{1-G_{d}\left(G_{f}\left(x_{i}\right)\right)}
$$

\section{Federated Learning for Privacy Protection}

Once an application-specific model is built, federated learning is performed iteratively within a group of devices of the same application. For instance, the smart devices shown in Fig. 1 can be a set of smart cameras, which may collect image or video data for different applications, such as for the purpose of product quality monitoring or worker detection and tracking. Assuming $x_{j}$ is the input samples of a data owner $c_{j}$, a CNN model that is adopted by the horizontal federated learning to achieve data privacy is trained locally by the data owner $c_{j}$ and represented by the hypothesis $l\left(x_{j}, \omega\right)$.

$$
l\left(x_{j}, \omega\right)=\mathrm{FC}\left(\operatorname{Pool}\left(\operatorname{Conv}\left(x_{j}, \omega_{\operatorname{Conv}}\right), \omega_{\text {Pool }}\right), \omega_{F C}\right)
$$

where $\operatorname{Conv}(*), \operatorname{Pool}(*)$ and $\mathrm{FC}(*)$ stand for the convolution layers, pooling layers and fully connected layers respectively and $\omega_{*}$ indicates the parameters of the corresponding layers.

The result of the hypothesis is predicted by a SoftMax classifier in the prediction layer. The hidden layer $G_{y}\left(R, \theta_{y}\right)$ stands for a function to learn a map from the extracted features $G_{f}\left(x_{i}, \theta_{f}\right)$ to the predicted label $y_{i}$.

$$
G_{y}\left(R, \theta_{y}\right)=\operatorname{SoftMax}\left(W_{y} R+b_{y}\right)
$$

where $\operatorname{SoftMax}(\mathbf{x})=\left[\frac{\exp \left(x_{k}\right)}{\sum_{j=1}^{|\mathbf{x}|} \exp \left(x_{j}\right) \exp }\right]_{k=1}^{|\mathbf{x}|}$.

Thus, the prediction loss according to a label classification output computing layer can be defined as follows.

$$
\mathcal{L}_{y}^{i}=\mathcal{L}_{y}\left(G_{y}\left(G_{f}\left(x_{i} ; \theta_{f}\right) ; \theta_{y}\right), y_{i}\right)
$$

where $\mathcal{L}_{y}\left(G_{y}\left(G_{f}\left(x_{i} ; \theta_{f}\right) ; \theta_{y}\right)\right.$ can be calculated by the negative log-probability of the correct label as $\log \frac{1}{G_{y}\left(G_{f}\left(x_{i}\right)\right)_{y_{i}}}$ [27]. This loss is designed to improve the discriminativeness of the classifier by minimizing the error rate between the predicted label and the real label.

In each iteration, the local parameters acquired in a local model will be encrypted and uploaded to the central server. The central server performs model aggregation by combining the received parameters based on Eq. (7).

$$
g_{m}(\omega)=\frac{1}{T} \sum_{j=1}^{T} \omega_{j}
$$

\section{EXPERIMENT AND ANALYSIS}

In this section we introduce the detailed experiment setup, and discuss the evaluation results in terms of the learning efficiency and accuracy of the proposed method.

\section{A. Dataset and Experiment Setup}

The proposed FTL-CDP framework is evaluated by adopting a generic object detection application as its base model generated by an existing application. This base model is subsequently applied to a different and more sophisticated application (i.e., pedestrian detection) in our experimental evaluation. The idea is to demonstrate that by using a model from a different application as the starting point, the proposed federated transfer learning can learn a new target application model in less time with less training data, while achieving comparable accuracy.

The generic object detection base model is trained with the YOLOv3 (hereafter referred to as YOLO) darknet architecture and COCO dataset. The COCO dataset offers 35 features and more than 118287 samples from 64115 subjects. The base model is stored at the central server and later shared with each individual smart device upon their request. At the smart device side, transfer learning is applied with the PETS2009 dataset, which contains 1900 images of crowds of pedestrians in a university campus setting, for emulating a typical crowd of workers within a warehouse setting.

To verify the performance for the proposed approach, the proposed FTL-CDP framework is evaluated against two 
state-of-the-art approaches, YOLO and the Siamese Convolutional Autoencoder (SCAE) model [28]. YOLO is selected to represent the case of a centralized $\mathrm{CNN}$-based approach, whereas SCAE is incorporated as a federated learning approach. The proposed FTL-CDP is compared with these two approaches to demonstrate its learning performance.

\section{B. Learning Efficiency Analysis}

One of the key advantages and contributions of the proposed FTL-CDP framework is its ability to facilitate faster learning time with limited training data. Therefore, we first evaluate the performance on training time and communication overheads along with the increasing number of samples ranging from 10 to 1000 , which is shown in Fig. 2. In all cases, a similar general trend can be observed, where the consumed time and communication overhead (in GB) increase linearly with the increased number of samples.

Referring to Fig. 2(a), it can be observed that the proposed FTL-CDP outperforms the other methods with the lowest processing time to achieve the same performance. For the case of 1000 samples, the YOLO model takes around 60 seconds to process, whereas the federated SCAE approach takes around 30 seconds and the proposed FTL-CDP takes only 9 seconds. This result indicates that federated learning based approaches (i.e., SCAE and FTL-CDP) can achieve more efficient training time in comparison with a centralized approach (i.e., YOLO). In addition, FTL-CDP achieves a better training time in comparison with the SCAE which only uses federated learning. This result shows that the transfer learning is improving the learning efficiency.

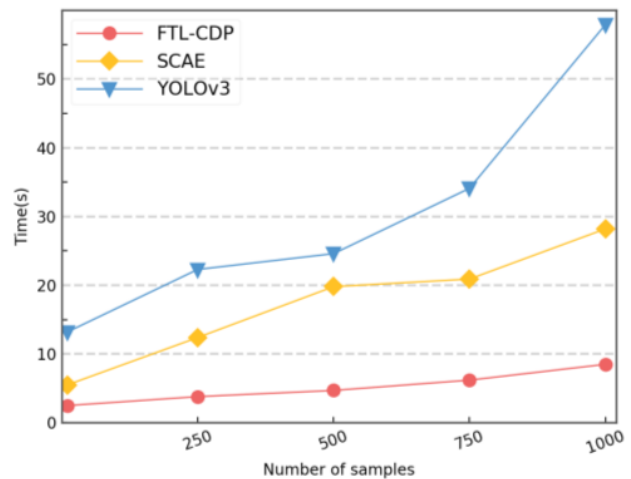

(a)

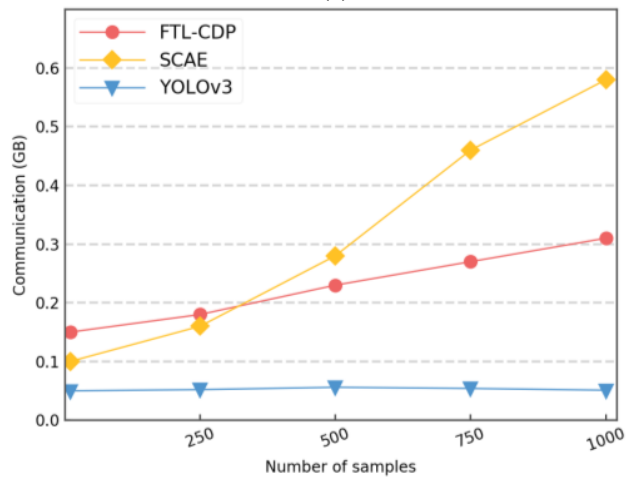

(b)

Fig. 2. (a) Training time and (b) communication overhead versus the number of training samples.

On the other hand, the federated learning based approaches have incurred more communication overheads in comparison with a local training model as shown in Fig. 2(b). The communication cost of YOLO is around 50MB communication overheads and remains relatively steady across different numbers of samples due to the fact that all the trainings are done locally. Initially, FTL-CDP incurs more communication overheads because of the sharing of the base model. As the number of samples increases, the federated SCAE becomes less efficient and results in higher communication overheads than FTL-CDP, which adopts transfer learning to achieve a more efficient learning.

Based on these results, we further analyze the difference in error rate convergence considering different sample sizes. The aim is to evaluate and demonstrate the performance of the proposed FTL-CDP in using less training data to achieve fast and accurate learning in comparison with the other two state-of-the-art methods. In this experiment, the federated learning based approaches contain two smart devices. The total number of samples processed by each method are 1000,5000 , or 10000 samples. In all three cases, a similar trend can be observed, where lower error rates can be achieved with more samples.

Referring to Fig. 3, it can be seen that all methods achieve comparable results after 1000 iterations. The federated learning based approaches achieve slightly better error rate in comparison with the centralized YOLO approach, especially in the case of small sample size (i.e., 1000 samples). However, it is interesting to compare the performances of different methods at 200 iterations. It can be observed that at 200 iterations, FTL-CDP achieves error rates of $0.12,0.1$, and 0.05 for sample size 1000,5000 , and 10000 respectively. In comparison, YOLO achieves error rates of 0.27 , $0.23,0.18$, and SCAE achieves error rates of $0.3,0.19,0.14$, respectively. This observation shows that, while federated learning based approach (i.e., SCAE) can eventually achieve a better error rate after 1000 iterations, it does not necessarily converge faster than a centralized learning approach.

In comparison, the proposed FTL-CDP makes use of the strength of transfer learning to achieve faster convergence and accurate learning even with small training size, which fulfills the need of the targeted smart manufacturing scenario. In addition, it is interesting to note that the proposed FTL-CDP achieves a more consistent learning with less fluctuation during the learning process, which indicates that transfer learning is providing a good base model to facilitate the learning of a target domain model.

\section{Classification Performance Analysis}

We go further to evaluate the classification accuracies of different methods. The aim is to demonstrate the difference in making use of the source and target domain dataset. We randomly select 10000 samples from the source domain (COCO) and 1000 samples from target domain (PETS2009) to emulate the scenario of lacking sufficient training samples in the target domain. Table 1 highlights the average classification results with their standard deviation for comparing the proposed FTL-CDP with YOLO and federated SCAE. The experiment results show that all the methods obtain a slightly worse performance in the target domain in terms of all the metrics including precision, recall, F1 score, AUC (Area Under the Curve), and IOU (Intersection Over Union). This is to be expected because the target domain has limited training samples. 


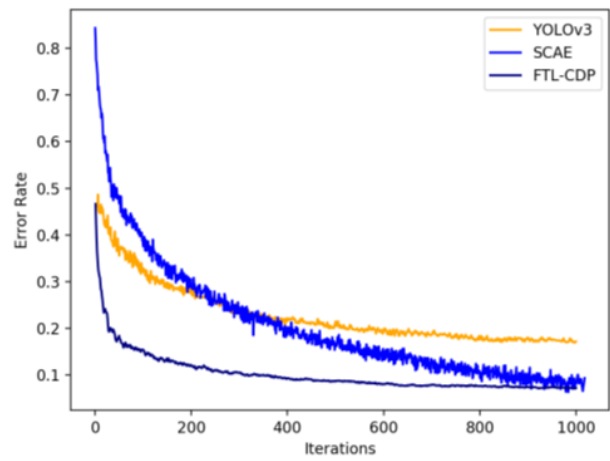

(a) Error Rate for 1000 samples

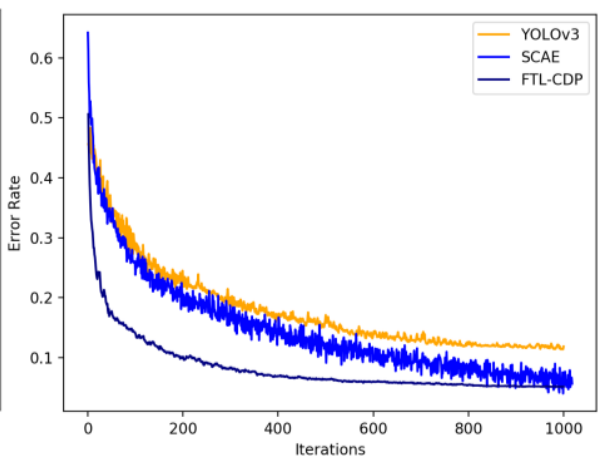

(b) Error Rate for 5000 samples

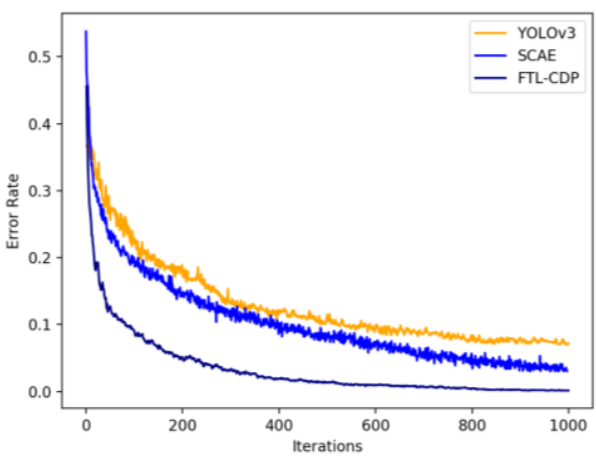

(c) Error Rate for 10000 samples

Fig. 3. Loss curve for different numbers of training samples and iterations.

TABLE 1. Classification ACCURACy ON Source (COCO) AND TARget (PETS2009) Domain

\begin{tabular}{|c|c|c|c|c|c|c|c|c|c|c|}
\hline \multirow{2}{*}{ Method } & \multicolumn{5}{|c|}{ Source Domain (General Object Dection) } & \multicolumn{5}{|c|}{ Target Domain (Pedestrian Detection) } \\
\hline & Precision & Recall & $\mathrm{F} 1$ & AUC & IOU & Precision & Recall & F1 & AUC & IOU \\
\hline YOLO & $.88 \pm .05$ & $.94 \pm .04$ & $.92 \pm .04$ & $.84 \pm .04$ & $.91 \pm .05$ & $.76 \pm .06$ & $.86 \pm .15$ & $.81 \pm .09$ & $.76 \pm .12$ & $.87 \pm .07$ \\
\hline SCAE & $.97 \pm .03$ & $.92 \pm .04$ & $.95 \pm .04$ & $.96 \pm .03$ & $.91 \pm .07$ & $.81 \pm .07$ & $.87 \pm .14$ & $.84 \pm .12$ & $.82 \pm .14$ & $.83 \pm .08$ \\
\hline FTL-CDP & $.97 \pm .04$ & $.95 \pm .03$ & $.95 \pm .03$ & $.94 \pm .03$ & $.93 \pm .05$ & $.89 \pm .05$ & $.87 \pm .12$ & $.88 \pm .08$ & $.89 \pm .09$ & $.90 \pm .06$ \\
\hline
\end{tabular}

In the source domain, it can be observed that the proposed FTL-CDP achieves either better or comparable classification performance in all the evaluation metrics in comparison with the federated SCAE. Both FTL-CDP and SCAE perform better than YOLO. This demonstrates that the deep learning model incorporating federated learning scheme can enhance the classification accuracy effectively by aggregating different sources of training samples.

Observing the results in the target domain, we note that the performance of FTL-CDP is significantly better than the others, with F1 score 0.88, AUC 0.89 and IOU 0.90. This result reflects the strengthen of the proposed FTL-CDP. By incorporating transfer learning, FTL-CDP outperforms the federated SCAE by achieving better classification accuracies with better consistency (i.e., less spread for every evaluation metric). These results suggested that our proposed method is able to support cross-domain applications in modern smart manufacturing, by incorporating the transfer learning scheme with federated learning architecture to tackle the limited labelled training samples for newly established applications.

\section{Cross-Domain Performance Analysis}

As mentioned previously, the main idea of this study is to make use of models from well-trained existing applications to facilitate the learning process of new cross-domain applications and to achieve a comparable learning result. Datasets from different domains have different distributions and features. Thus, we go further to evaluate and demonstrate the effectiveness of the adopted domain adaptation approach in extracting discriminative features in both the source and target domains. The extracted features visualized through PCA (Principal Component Analysis) are shown in Fig. 4.

Following the proposed framework, a base model of an existing application is available on the central server. In this experiment, the base model is trained using the COCO dataset.
Similar to the previous experiments, two smart devices are instantiated to perform the federated transfer learning upon receiving the base model. In can be observed in Fig. 4(a) that the base model trained using the COCO dataset extracts features that can effectively discriminate data into several clusters in the source domain. Based on the received base model, each smart device performs cross-domain adaptation process to train a target domain model individually using limited target domain data randomly selected from the PETS2009 dataset. Fig. 4(b) and (c) show the extracted features of smart device one and two respectively. Since both devices are trained using randomly selected data from PETS2009, the data can be considered as independently and identically distributed from the same target application. This is reflected by the similar distribution shown in Fig. 4(b) and (c). The transferred models for the target domain show reasonable discriminative ability in forming several clusters. This result demonstrates that the transferred target domain model is able to provide a reasonable starting point to speed up the training process.

After the transfer learning process, federated learning is used to further strengthen the target domain models by aggregating local models from the two smart devices to iteratively train a better target domain model. Fig. 4(d) shows clearly that after the federated learning, the extracted features can more effectively discriminate the data samples with better separated clusters (e.g., on the bottom right corner). This result demonstrates the strength of the proposed framework, which adopts transfer learning to effectively transfer a base model from an existing application to establish a new target domain model with limited training data, and then apply federated learning to aggregate information from individual smart devices to improve model accuracy while protecting data privacy. 


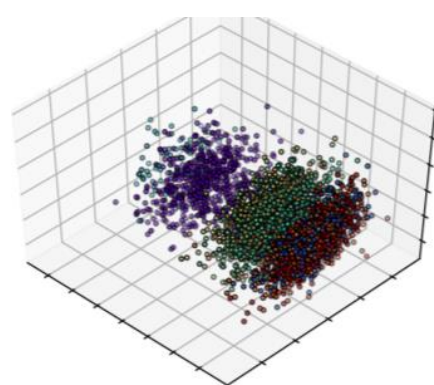

(a)

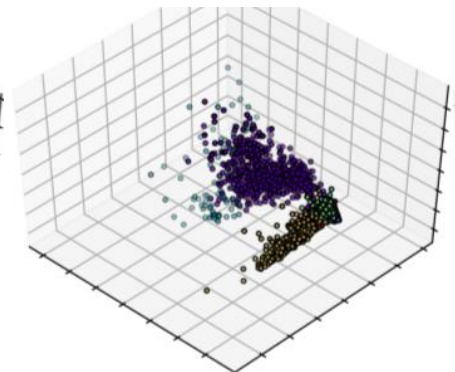

(b)

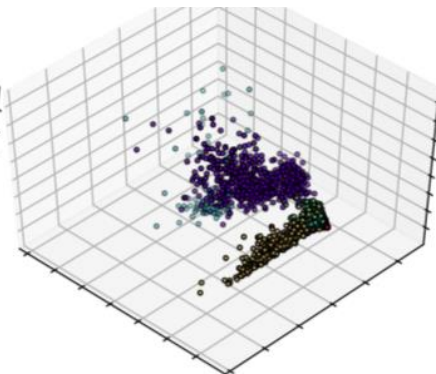

(c)

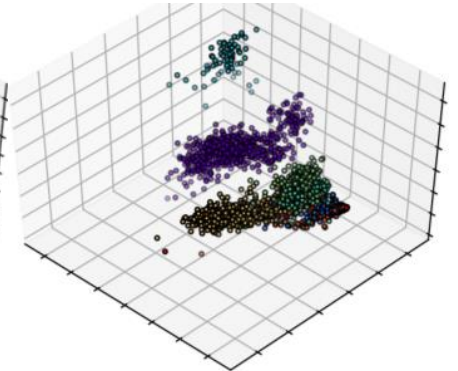

(d)

Fig. 4. Discriminative capability of the extracted features by PCA visualization for (a) features extracted in the source domain with 10000 samples, (b) features extracted by device 1 after cross-domain transfer learning with 1000 samples, (c) features extracted by device 2 after cross-domain transfer learning with 1000 samples, and (d) features extracted after federated learning with device 1 and 2.

\section{CONCLUSION}

Modern smart manufacturing applications are highly customizable, and therefore require machine intelligence techniques to be quickly adaptable to different application domains with limited application data. Very often, application-specific data needs to be protected from each individual data owner and the cloud server, to avoid unnecessary data leak on confidential product or personal information related to a manufacturing process. While existing federated learning approach is able to protect data privacy, its learning efficiency and accuracy may be limited, especially with limited application-specific data. In this paper, a federated transfer learning framework, FTL-CDP, was proposed targeting cross-domain predictions in Industry 4.0 applications. Existing base models and domain knowledge could be shared using the federated learning architecture, while individual smart devices could adopt the base model and update the model using transfer learning with local application-specific data. The proposed approach is evaluated against the state-of-the-art centralized approach YOLO and a federated Siamese Convolutional Autoencoder (SCAE) approach, and the comparison results demonstrated better learning efficiency and accuracy of the proposed FTL-CDP in a real-world pedestrian detection application.

\section{ACKNOWLEDGMENT}

The work was supported in part by the National Natural Science Foundation of China under Grant 72088101, 72091313, and 62072171, in part by the National Key R\&D Program of China under Grants 2017YFE0117500, 2019YFE0190500, and 2019GK1010, and in part by the Natural Science Foundation of Hunan Province of China under Grants 2019JJ40150.

\section{REFERENCES}

[1] J. Wang, J. Hu, G. Min, A. Y. Zomaya and N. Georgalas, "Fast Adaptive Task Offloading in Edge Computing Based on Meta Reinforcement Learning," IEEE Transactions on Parallel and Distributed Systems, vol. 32, no. 1, pp. 242-253, 1 Jan. 2021.

[2] Z. Qin et al., "Learning-Aided User Identification Using Smartphone Sensors for Smart Homes," IEEE Internet of Things Journal, vol. 6, no. 5, pp. 7760-7772, Oct. 2019.

[3] Z. Chen, J. Hu, G. Min, A. Y. Zomaya and T. El-Ghazawi, "Towards Accurate Prediction for High-Dimensional and Highly-Variable Cloud
Workloads with Deep Learning," IEEE Transactions on Parallel and Distributed Systems, vol. 31, no. 4, pp. 923-934, 1 April 2020.

[4] E. Nowroozi, A. Dehghantanha, R. M. Parizi and K. K. R. Choo, "A Survey of Machine Learning Techniques in Adversarial Image Forensics," in Computers \& Security, vol. 100, Jan. 2021.

[5] Y. Wu, "Cloud-Edge Orchestration for the Internet-of-Things: Architecture and AI-Powered Data Processing," IEEE Internet of Things Journal, Aug. 2020, doi: 10.1109/JIOT.2020.3014845.

[6] Z. Qin, G. Huang, H. Xiong, Z. Qin and K. -K. R. Choo, "A Fuzzy Authentication System Based on Neural Network Learning and Extreme Value Statistics," IEEE Transactions on Fuzzy Systems, vol. 29, no. 3, pp. 549-559, March 2021.

[7] Q. Yang, Y. Liu, T. Chen, Y. Tong, "Federated machine learning: Concept and applications," ACM Transactions on Intelligent Systems and Technology (TIST), vol. 10, no. 2, pp. 1-19, 2019.

[8] S. K. Lo, Q. Lu, C. Wang, H. -Y. Paik and L. Zhu, "A Systematic Literature Review on Federated Machine Learning: From a Software Engineering Perspective," ACM Computing Survey, vol. 54, no. 5, pp.1-39, 2021.

[9] Y. Wu, H.-N. Dai and H. Wang, "Convergence of Blockchain and Edge Computing for Secure and Scalable IIoT Critical Infrastructures in Industry 4.0," IEEE Internet of Things Journal, vol. 8, no. 4, pp. 2300-2317, Feb. 2021

[10]W. Zhang et al., "Blockchain-Based Federated Learning for Device Failure Detection in Industrial IoT," in IEEE Internet of Things Journal, vol. 8, no. 7, pp. 5926-5937, 2021.

[11]K. Weiss, T. M. Khoshgoftaar, D. Wang, "A survey of transfer learning," Journal of Big data, vol. 3, no. 1, pp. 1-40, 2016.

[12]M. Song et al., "Analyzing User-Level Privacy Attack Against Federated Learning," IEEE Journal on Selected Areas in Communications, vol. 38, no. 10, pp. 2430-2444, Oct. 2020.

[13]L. Lyu et al., "Towards Fair and Privacy-Preserving Federated Deep Models," IEEE Transactions on Parallel and Distributed Systems, vol. 31, no. 11, pp. 2524-2541, 1 Nov. 2020.

[14]H. Zhu and Y. Jin, "Multi-Objective Evolutionary Federated Learning," IEEE Transactions on Neural Networks and Learning Systems, vol. 31, no. 4, pp. 1310-1322, April 2020.

[15]Y. Lu, X. Huang, Y. Dai, S. Maharjan and Y. Zhang, "Blockchain and Federated Learning for Privacy-Preserved Data Sharing in Industrial IoT," IEEE Transactions on Industrial Informatics, vol. 16, no. 6, pp. 4177-4186, June 2020.

[16]M. Hao, H. Li, X. Luo, G. Xu, H. Yang and S. Liu, "Efficient and Privacy-Enhanced Federated Learning for Industrial Artificial Intelligence," IEEE Transactions on Industrial Informatics, vol. 16, no. 10, pp. 6532-6542, Oct. 2020.

[17]W. Zhang et al., "Dynamic Fusion-based Federated Learning for COVID-19 Detection," IEEE Internet of Things Journal, doi: 10.1109/JIOT.2021.3056185.

[18]M. Fang, J. Yin, X. Zhu and C. Zhang, "TrGraph: Cross-Network Transfer Learning via Common Signature Subgraphs," IEEE Transactions on 
Knowledge and Data Engineering, vol. 27, no. 9, pp. 2536-2549, Sept. 2015.

[19]C. Sun, M. Ma, Z. Zhao, S. Tian, R. Yan and X. Chen, "Deep Transfer Learning Based on Sparse Autoencoder for Remaining Useful Life Prediction of Tool in Manufacturing," IEEE Transactions on Industrial Informatics, vol. 15, no. 4, pp. 2416-2425, April 2019.

[20]Q. Wu et al., "Online Transfer Learning with Multiple Homogeneous or Heterogeneous Sources," IEEE Transactions on Knowledge and Data Engineering, vol. 29, no. 7, pp. 1494-1507, July 2017.

[21] X. Li, W. Zhang, Q. Ding and X. Li, "Diagnosing Rotating Machines With Weakly Supervised Data Using Deep Transfer Learning," IEEE Transactions on Industrial Informatics, vol. 16, no. 3, pp. 1688-1697, March 2020.

[22]L. Yang, L. Jing, J. Yu and M. K. Ng, "Learning Transferred Weights From Co-Occurrence Data for Heterogeneous Transfer Learning," IEEE Transactions on Neural Networks and Learning Systems, vol. 27, no. 11, pp. 2187-2200, Nov. 2016.

[23]Y. Liu, Y. Kang, C. Xing, T. Chen and Q. Yang, "A Secure Federated Transfer Learning Framework," IEEE Intelligent Systems, vol. 35, no. 4, pp. 70-82, 1 July-Aug. 2020.

[24] S. Sharma, C. Xing, Y. Liu and Y. Kang, "Secure and Efficient Federated Transfer Learning," 2019 IEEE International Conference on Big Data (Big Data), Los Angeles, CA, USA, 2019 pp. 2569-2576.

[25]Y. Chen, X. Qin, J. Wang, C. Yu and W. Gao, "FedHealth: A Federated Transfer Learning Framework for Wearable Healthcare," IEEE Intelligent Systems, vol. 35, no. 4, pp. 83-93, 1 July-Aug. 2020.

[26]C. Ju, D. Gao, R. Mane, B. Tan, Y. Liu and C. Guan, "Federated Transfer Learning for EEG Signal Classification," 42nd Annual International Conference of the IEEE Engineering in Medicine \& Biology Society (EMBC), Montreal, QC, Canada, 2020, pp. 3040-3045.

[27]Y. Ganin et al., "Domain-adversarial training of neural networks," The Journal of Machine Learning Research, vol. 17, no. 1, pp. 1-35, 2016.

[28]D. Droghini, S. Squartini, E. Principi, L. Gabrielli and F. Piazza, "Audio Metric Learning by Using Siamese Autoencoders for One-Shot Human Fall Detection," in IEEE Transactions on Emerging Topics in Computational Intelligence, vol. 5, no. 1, pp. 108-118, Feb. 2021.

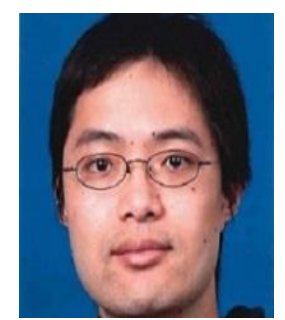

Kevin I-Kai Wang (M'04) received the Bachelor of Engineering (Hons.) degree in Computer Systems Engineering and $\mathrm{PhD}$ degree in Electrical and Electronics Engineering from the Department of Electrical and Computer Engineering, the University of Auckland, New Zealand, in 2004 and 2009 respectively. He is currently a Senior Lecturer in the Department of Electrical, Computer, and Software Engineering, the University of Auckland. He was also a research engineer designing commercial home automation systems and traffic sensing systems from 2009 to 2011. His current research interests include wireless sensor network based ambient intelligence, pervasive healthcare systems, human activity recognition, and distributed intelligence and automation.

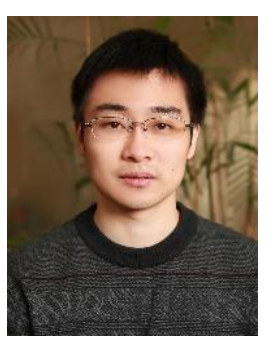

Xiaokang Zhou (M'12) is currently an associate professor with the Faculty of Data Science, Shiga University, Japan. He received the Ph.D. degree in human sciences from Waseda University, Japan, in 2014. From 2012 to 2015, he was a research associate with the Faculty of Human Sciences, Waseda University, Japan. $\mathrm{He}$ also works as a visiting researcher in the RIKEN Center for Advanced Intelligence Project (AIP), RIKEN, Japan, since 2017. Dr. Zhou has been engaged in interdisciplinary research works in the fields of computer science and engineering, information systems, and social and human informatics. His recent research interests include ubiquitous computing, big data, machine learning, behavior and cognitive informatics, cyber-physical-social systems, cyber intelligence and security. Dr. Zhou is a member of the IEEE CS, and ACM, USA, IPSJ, and JSAI, Japan, and CCF, China.

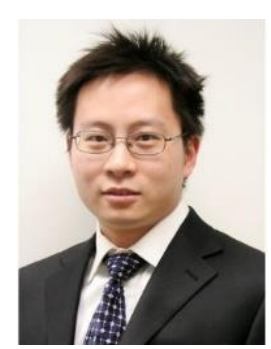

Wei Liang (M'19) received his M.S. and Ph.D. degrees in Computer Science from Central South University in 2005 and 2016. From 2014 to 2015, he was a researcher with the Department of Human Informatics and Cognitive Sciences, Waseda University, Japan. $\mathrm{He}$ is currently working at Base of International Science and Technology Innovation and Cooperation on Big Data Technology and Management, Hunan University of Technology and Business, China. His research interests include information retrieval, data mining, and artificial intelligence. He has published more than 20 papers at various conferences and journals. Dr. Liang is a member of the IEEE CS, and CCF, China.

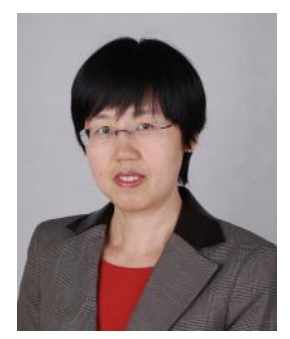

Zheng Yan (M'06, SM'14) received the BEng degree in electrical engineering and the MEng degree in computer science and engineering from the Xi'an Jiaotong University, Xi'an, China in 1994 and 1997, respectively, the second MEng degree in information security from the National University of Singapore, Singapore in 2000 , and the licentiate of science and the doctor of science in technology in electrical engineering from Helsinki University of Technology, Helsinki, Finland. She is currently a professor at the Xidian University, Xi'an, China and a visiting professor at the Aalto University, Espoo, Finland. Her research interests are in trust, security, privacy, and security-related data analytics. Prof. Yan serves as a general or program chair for $30+$ international conferences and workshops. She is a steering committee co-chair of IEEE Blockchain international conference. She is also an associate editor of many reputable journals, e.g., IEEE Internet of Things Journal, Information Sciences, Information Fusion, JNCA, IEEE Access, SCN, etc.

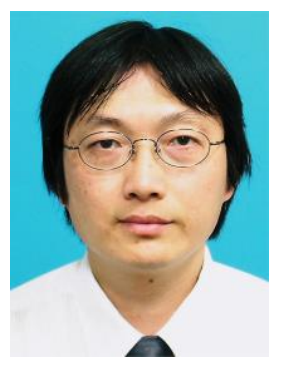

Jinhua She (M'94, SM'08, FM'21) received his B.S. degree in engineering from Central South University, Changsha, China in 1983, and his M.S. and Ph.D. degrees in engineering from the Tokyo Institute of Technology, Tokyo, Japan in 1990 and 1993, respectively. In 1993, he joined the School of Engineering, Tokyo University of Technology, Tokyo, where he is currently a Professor with the Department of Mechanical Engineering. His research interests include the application of control theory, repetitive control, process control, Internet-based engineering education, and assistive robotics. Dr. She is a member of the Society of Instrument and Control Engineers, the Institute of Electrical Engineers of Japan, the Japan Society of Mechanical Engineers, and the Asian Control Association. He was the recipient of the International Federation of Automatic Control (IFAC) Control Engineering Practice Paper Prize in 1999 (jointly with M. Wu and M. Nakano). 